\title{
Transnumeralität im Türkischen
}

\author{
Türkçede Belirtilmemiş Sayı
}

Nihal DURMUŞ*

Zusammenfassung: In vielen indoeuropäischen Sprachen, wie dem Deutschen, unterliegen die meisten Nomina der semantischen Unterscheidung [ \pm zählbar]. Traditionell werden diese in zwei klar abgrenzbare, wenn auch nicht "disjunkte Subkategorien" (Krifka, 1989) eingeteilt, und zwar in "Individualnomina" (count nouns) und "Massennomina" (mass nouns). Massennomina werden weiter unterteilt in Stoff-und Kollektivnomina, wobei sich Letztere noch weiter untergliedern lassen in "Gruppen-und Genuskollektiva" (Leisi, 1971). Auch bei Abstrakta tritt diese Unterscheidung zwischen Massen-und Individualnomina auf. Die meisten Numerussysteme indoeuropäischer Sprachen unterscheiden zwischen zwei Numeri, Singular („eine Einheit“) und Plural („mehr als eine Einheit“). Im grammatischen System der türkischen Sprache scheint prima facie die Kategorie des Numerus ebenfalls kodiert zu sein, wobei zwischen zwei Numeri, Singular und Plural, unterschieden wird. Ferner macht es den Anschein, dass sich Nomina, gemäß der semantischen Unterscheidung [ \pm zählbar], ebenso in Individual- und Massennomina untergliedern lassen. Im Gegensatz zum Deutschen scheint die türkische Sprache allerdings eine Besonderheit aufzuweisen, die sich in der "unterschiedlichen morpho-syntaktischen Handhabung der Kategorie 'Vielzahl' bei der Verwendung von Pluralformen widerspiegelt" (Johanson/Rehbein, 1999). Diese Besonderheit der türkischen Sprache wird dem linguistischen Phänomen "Transnumeralität" zugeordnet, was den Gegenstand der folgenden Untersuchung bildet.

Schlüsselwörter: Türkisch, Semantik, Markierung der Kategorie Numerus, Transnumeralität

Özet: Almanca gibi birçok Hint-Avrupa dillerinde, isimler [ \pm sayılabilir] anlambilimsel ayrıma tabidir. Dilbilimsel açıdan, isimler ikiye, yani sayı ve kütle isimler şeklinde ayrılırlar (Krifka, 1989). Kütle isimleri ayrıca ikiye ayrılırlar, malzeme ve kolektif isimler olarak. Kolektif isimler yine aynı şekilde ikiye ayrılır: grup ve cins isimler olarak (Leisi, 1971). Kütle ve sayı isimleri arasındaki bu ayrım aynı zamanda soyut isimlerin grup içerisinde de bulunmaktadır. Birçok Hint-Avrupa dillerinin sayı sistemlerin çoğu iki sayı, tekil ("tek bir birim") ve çoğul ("birden fazla birim") arasında ayrım yapmaktadır. Türk dilinin gramer sisteminde sayı kategorisi aynı şekilde, yanı tekil ve çoğul olarak, kodlanmış gibi görünmektedir. Bunun yanı sıra, Türk dilinde isimler, sayı ve kütle isimleri olarak [ \pm sayılabilen] anlambilimsel ayrıma tabi olduğu gibi görünmektedir. Ancak Alman dilinin aksine, Türk dili "çoğul formlarının kullanımda 'çokluk' farklı morfolojik-sözdizimsel uygulamasını yansıtan" bir özelliğe sahiptir (Johanson/Rehbein, 1999). Türk dilinin bu özelliği "transnumerality" dilsel olgusuna atanır ve bu da araştırmanın konusudur.

Anahtar sözcükler: Türkçe, anlambilim, sayı işaretlemesi, belirtilmemiş sayı

\section{Einleitung}

Bevor es im Hauptteil der Untersuchung um diese Besonderheit der türkischen Sprache gehen soll, gilt es einen Blick auf die Wortklasseneinteilung im Türkischen zu werfen. Grundsätzlich

\footnotetext{
* Öğr. Gör., Akdeniz Üniversitesi, Batı Dilleri ve Edebiyatları Bölümü, Antalya, nihaldurmus@akdeniz.edu.tr
} 
unterscheidet man im grammatischen System der türkischen Sprache zwischen zwei lexikalischen Kategorien: Nomen (isim) und Verben (eylem, fiil) (Csató/Johanson, 1998, 208). Diese Einteilung basiert auf morphologischen Grundsätzen, da einige Suffixe nur mit Nomen und andere nur mit Verben kombinierbar sind (Schröder, 1999, 22). Abgesehen von einigen abgeleiteten und entlehnten Adjektiven, z. B. demokratik „demokratisch“ (Johanson, 1990, 187), werden Nomen und Adjektive (sıfat) morphologisch nicht unterschieden:

[The adjective] may receive nominal morphology, be the head of a noun phrase and the argument of a verb. [...] words expressing relational qualities like iyi 'good', and kötü 'bad' may function both as adjectives as in (1) and as nouns, as in (2): (1) iyi bir adam [good a man] 'a good man'; (2) kötü-yü iyi-yi ayırt ed-ecek-sin [bad-ACC good-ACC distinguish-FUT-2SG] 'You will distinguish (the) good and (the) bad (Schröder, 1999, 22).

Eine Erklärung für diese Art der Wortklasseneinteilung im Türkischen findet sich bei Grönbech (1979), der Folgendes hierzu bemerkt:

The reason for this is that one cannot separate them conceptually. Nouns do not denote a being or a thing, but rather the indefinite concept of these, defined neither substantively nor adjectivally. This means that one speaks of a concept without stressing whether it is to be envisioned as a thing or as a quality. The first consequence of this nominal concept is that originally there was no linguistic expression of the division between singular and plural in the inflection. The oldest language had no plural forms. /.../ The fact that all Turkic languages have a plural suffix today proves that this nominal concept was displaced in time. /.../ a Turkic noun is neither a substantive nor an adjective, but namely both at the same time (Grönbech, 1979, 24; vgl. Übers. v. Akalın, 1995, 50-70).

\section{Transnumeralität im Türkischen}

Im Türkischen bildet der Ausdruck der Quantität einen Teil der Grammatik, wobei zwischen zwei Numeri unterschieden wird, wie Kornfilt $(1997,265)$ feststellt: "Turkish does have a number marking system in nouns. The system has a main singular-plural opposition". Folgende Beispiele sollen diesen minimalen Numeruskontrast verdeutlichen:

1) İstanbulun sokakları içerisinde bir yerde, bizimkine benzeyen bir başka evde, her şeyiyle benim benzerim, ikizim, hatta tıpatıp aynım bir başka Orhan'ın yaşadığına çocukluktan başlayarak uzun yıllar aklımın bir kössesiyle inandım (Pamuk, 2004, 9).

[Istanbul-POSS Straße-PL-POSS innerhalb-LOK indef. Art. Platz-LOK, wie unser ähnlich sein indef. Art. anderes Haus-LOK jede-POSS Ding mir ähnlich Zwilling sogar Ebenbild indef. Art. Orhan leben von Kindheit anfangen-AORIST-PART. lang Jahr-PL Gedanke-POSS indef. Art. Ecke glauben-PRÄT.].

„Als Kind wurde ich lange den Gedanken nicht los, irgendwo in Istanbul, in einem Haus, wie dem unseren, müsse noch ein zweiter Orhan leben, ein Ebenbild von mir, ein Zwilling, ein zweites Selbst“ (Meier, 2003, 9). 
2) En gizli giyecek şeylerden yüzlerinin peçesine, eldivenlerinin rengine, mendillerinin işlemesine varıncaya kadar öyle bir güzel ve benzersiz zevk egemen olurdu ki sadelikleriyle birlikte en özenilmiş, en üzerinde durulmuş süsleri bayağılı̆̆a indirirdi (Uşaklıgil, 1987, 20).

[Superlativpartikel geheim Kleidung Ding-PL-LOK Gesicht-PL-POSS Schleier-POSS Handschuh-PL-POSS Farbe-POSS, Taschentuch-PLPOSS Stickerei-POSS kommen-AORIST-PART bis so NRL schön und ohne gleichen Geschmack vorherrschen sein-PRÄT dass Schlichtheit-PL zusammen Superlativpartikel Sorgfalt-walten-PERF Superlativpartikel darüber-LOK bleiben-PERF Schmuck-PL-POSS Banalität herabsetzenPRÄT]

„Angefangen bei den intimsten Kleidungsstücken bis zu ihren Gesichtsschleiern, der Farbe ihrer Handschuhe und der Stickerei ihrer Taschentücher herrschte ein höchst erlesener und außergewöhnlicher Geschmack, der im Einklang mit seiner Schlichtheit die überaus sorgfältig ausgeführten Handarbeiten ganz alltäglich erschienen ließ“ (Riemann, 2007, 18).

Zur Entwicklung des Pluralsuffixes -IAr bemerkt Grönbech (1936, 61f.) Folgendes: "Historically ler/-lar is classified as a collective suffix, which in Old Turkish (sixth/seventh century) only occurred with words referring to sovereigns and older female relatives. Later it was first employed to mark the (collective) plurality of nouns denoting animate entities, then of nouns in general" (Vgl. Georg (1989), "Some thoughts on the etymology of the Turkic pluralsuffix -lar-/ler” und Kowalski (1936), „Zur semantischen Funktion des Pluralsuffixes -LAR, -LÄR in den Türksprachen“. In (2) beziehen sich die mit -lAr markierten Nomen auf eine Pluralität von Entitäten, der Ausdruck bir evde ist in Kombination mit dem indefiniten Artikel in (1) singularisch verwandt. Daraus lässt sich eine erste Annahme ableiten: (1) „Enthält der Kontext und/ oder die Sprechsituation einen Hinweis(e) auf Individuen, Exemplare, so dass das [Nomen] mit -lAr in Beziehung zu außersprachlichen Individuen/Exemplaren gesetzt ist, dann bezeichnet das Pluralmorphem die Mehrzahl dieser Individuen/Exemplare“ (Götz, 1980, 383), ggf. mit der zusätzlichen Bedeutungskomponente „Sortigkeit“. Ein interessantes Beispiel zur Numerusverwendung im Türkischen führt Götz $(1980,384)$ an:

3) Zenginler yoksulları düşünmelidir.

[Reich-PL Arm-PL-AKK denken-3.P.PL-FAKT]

„Die Reichen müssen an die Armen denken“.

Zenginler und yoksullar beziehen sich auf keine bestimmten außersprachlich gegebenen Individuen, die Nomen ,zengin ‘der Reiche' und yoksul 'der Arme' sind Gattungsnamen. Durch die Suffigierung von -ler-/-lar- erhalten sie die Bedeutung 'alle Reichen', 'die Reichen schlechthin' etc. In Verbindung mit einem Gattungsnamen hat der Plural die Bedeutung 'Totalität'“ (Götz, 1980, 383). Betrachten wir ein weiteres Beispiel, das einen weiteren Wesenszug des Plurals im Türkischen verdeutlicht:

4) Bir vakitler yalının pencerelerinden taşan şenlik eğlence sesleri hâlâ rıhtımın taşlarını yalayan suların ezgili seslerinde gizli, geceleri Boğaz'ın suları bir zamanlar buradan topladıkları sevinç parıltısının hâlâ kalan pırıltılarıyla ışıklı sanılır (Uşaklıgil, 1987, 13).

[Indef. Art. Zeit-PL Villa-AKK Fenster-PL-LOK ausströmen-PART Amüsement Klang-PL noch immer Mole-AKK Stein-PL-AKK lecken- 
PART Wasser-PL-AKK Melodie Klang-PL-LOK geheim Nacht-PLAKK Meerenge-AKK Wasser-PL-AKK indef. Art. Zeit-PL hier-LOK aufhäufen-PRÄT-PL-AKK Freude Glanz-AKK-POSS immer noch bleiben Glanz-PL Licht meinen]

„Die heitere Harmonie, die einstmals aus den Fenstern dieser Ufervilla nach draußen gedrungen war, scheint noch immer fortzuwirken in der Melodie der Wellen, die an den Steinen der Uferbefestigung lecken“ (Riemann, 2007, 9).

„Wo das Merkmal 'zählbar' nicht gegeben ist, lassen sich die Einzelbedeutungen wie Extensität, Intensität, Hyperbel oder Metapher feststellen“ (Götz, 1980, 384), wie auch suların und bir zamanlar in Beispiel (4) verdeutlichen. In diesem Zusammenhang lässt sich auf Kononov (1956, § 87; zit. nach Götz, 1980, 384f.) verweisen, der Folgendes anmerkt:

kar 'Schnee' - karlar 'Schneemassen'; martın ortası 'Mitte März' martın ortalarl 'So um Mitte März' (extensive Bedeutung); Afiyet olsun! 'Guten Appetit!' - Afiyetler olsun! 'Einen recht guten Appetit!'; Kana boyandl 'Er war blutüberströmt' - Kanlara boyandl 'Er war ganz und gar mit Blut überströmt' (intensive Bedeutung); kaç defa ölümlerden kurtuldum, neler çektim, biliyor musunuz? 'Wißt Ihr (eigentlich), wie viele Male ich aus Todesängsten (hyperbolisch) gerettet worden bin, was ich alles durchgemacht habe?', [...]; Beşyüz ev '500 Häuser' - Beşyüz evler (Name eines Stadtviertels vor den Toren Istanbuls. Das Zahlwort '500' im Kontext des Plurals ist 'verfremdet' und damit seines Zahlwertes verlustig gegangen $=$ Metapher $!)($ Kononov, 1956, §87).

Sehen wir uns einige weitere Beispiele nach Götz $(1980,382)$ an, die den Ausdruck der Quantität im Türkischen veranschaulichen:

5) Tersanede gemi yapılır ve tamir edilir.

[Werft-LOK Schiff bauen-AOR und reparieren-AOR]

„Auf einer/der Werft/auf Werften werden Schiffe gebaut und repariert“.

6) Gemi bu tersanede yapılmış ve tamir edilmiştir.

[Schiff diese Werft-LOK-miş-Vergangenheit/Pluperfekt]

„Das Schiff wurde auf dieser Werft gebaut und repariert“.

7) Almanya'da kitap pahalıdır.

[Deutschland-LOK Buch teuer-PRÄS.]

„In Deutschland sind Bücher teuer“.

8) Almanya'da satın aldı ̌̆ım kitap pahalıdır.

[Deutschland-LOK kaufen-AKK Buch teuer-PRÄS.]

„Das von mir in Deutschland gekaufte Buch ist teuer“.

In Beispielsatz (5) werden die Nomen tersane und gemi als Gattungsnamen verwendet (Götz, 1980, 382). In (6) stehen gemi und tersane im Singular. Im Beispielsatz (7) handelt es sich im Falle von kitap um einen Gattungsnamen (ebd.). In (8) ist das Nomen kitap ein singularisches Nomen. Daraus leitet sich eine weitere Annahme ab: „Im Singular wird nur dann das Individuum/das Exemplar einer Gattung bezeichnet, wenn der Kontext und/oder die Sprechsituation durch bestimmte Signale auf ein - außersprachliches - Individuum oder Einzelding hinweisen“ (Götz, 1980, 384). Mit dem Nomen „im Singular wählt der Sprecher eine Gattung“ aus, und 
betrachtet es ohne konkrete Konturen (ebd.). So sind gemi und tersane in (6) durch die Sprechsituation und das deiktische Pronomen bu im Sinne von Einzel determiniert, in Satz (8) wird durch die „attributive Ergänzung“ satın aldı̆̆ım der Singular kitap in Beziehung zu dem außersprachlich gegebenen Exemplar „Buch“ gesetzt (ebd., 383). Satz (5) und (7) enthalten keine derartigen Signale, weshalb es sich bei den Singularia um Gattungsbegriffe handelt (ebd.). Wie lassen sich folgende Beispiele nach Götz $(1989,383)$ erläutern?

9) Türkiye'de arsa alacă̆ım.

[Türkei-LOK Grundstück kaufen-FUT.1.P-SG].

10) Türkiye’de bir arsa alacă̆ım.

[Türkei-LOK ein Grundstück kaufen-FUT.1.P-SG].

Das Beispiel unter (9) kann je nach Sprechsituation entweder als „Ich werde in der Türkei ein Grundstück kaufen“, oder „Ich werde in der Türkei Grundstücke kaufen“ wiedergegeben werden, d. h. dieser Satz beinhaltet eine Komponente der Vagheit bzw. Ambiguität, da das Nomen arsa numerusindifferent ist (Götz, 1980, 383). Satz (10) bedeutet „Ich werde in der Türkei ein Grundstück kaufen“. Das unbetonte bir schränkt die Bedeutung des Gattungsnamens arsa im Sinne von „ein beliebiger Vertreter der Gattung arsa“ ein (ebd., 383). Das unbetonte bir ist also - im Gegensatz zum betonten Numerale bir - ein „Restriktionssignal“ (ebd.), doch mehr dazu weiter unten. Auf dieser Basis lässt sich eine dritte Annahme ableiten: „Ein Singular in Verbindung mit unbetontem bir ist stets Gattungsbegriff, ebenso der Singular als Modifikator zu einem Verbum, wobei das als Modifikator fungierende Substantiv im Akkusativ steht“ (ebd.). Wie schon das Beispiel unter (10) verdeutlicht, findet man im Türkischen Sätze, in denen Nomen scheinbar in „transnumeralisierender” Funktion auftreten:

11) Ve çocuklar ağzına kadar kuş dolu kafesleriyle yorgun, umutları kırılmış, bu kadar kuşu ne yapacaklarını bilemeden evlerine geri dönüyorlar (Kemal, 1978, 14).

[Und Kind-PL Mund-POSS bis Vogel gefüllt Käfig-PL-AKK-POSSKOM müde, Hoffnung-POSS-3.P kaputt-EVID, dies so viel Vogel-AKK was machen-FUT-3.PL wissen-NEG-PRÄT-3.P Haus-PL-POSS-3.PL zurück gehen-PRÄS-PL-3.P].

„Müde und enttäuscht kehren die Kinder mit den vollen Käfigen heim, ratlos, weil sie nicht wissen, was sie mit all den Vögeln anfangen sollen" (Bischoff, 1984, 14).

12) Kuşçular yüzlerce kuş arasından birkaç iyicesini, kuş meraklılarına çok pahalı ya satmak için alıp kalanını çocuklara gerisini geri veriyorlar (Kemal, 1978, 13f.).

[Vogelhändler-PL hundert-SUF-ADVER Vogel zwischen-POSS-ABL einige gut-SUF-ADVER Vogel Liebhaber-SUF-TEILM-PL-AKK sehr teuer-SUF-ADVER verkaufen deshalb kaufen-SUF-VERBALADVER übrigbleiben-AKK Kind-PL-AKK zurückbleiben-AKK zurückgebenPRÄS-3.P-PL].

„Die Vogelhändler dort suchen aus Hunderten von Vögeln nur die besten und schönsten heraus, um sie zu sehr hohen Preisen an Liebhaber zu verkaufen, und geben die restlichen zurück“ (Bischoff, 1984, 14).

Basierend auf Hengevelds (1992a) Wortklasseneinteilung ist davon aus zugehen, dass die türkische Sprache dem flexiblen Sprachtyp angehört, genauer dem "Typ 2 (V-N/A)", in der 
/.../ reference is made to the function(s) that a lexical item can fulfill without having to resort to special grammatical measures. Thus one can distinguish between lexical items that (without any special measures) can be used as the head of a referring expression (NP or term phrase), $i$. e. nouns, and lexical items that can be used (without having to resort to any special measures) to modify the head of a term, i.e. adjectives (Hengeveld, 1992a; zit. nach Rijkhoff, 2002, 16).

Hengeveld (1992a) unterscheidet insgesamt sieben Sprachtypen, darunter: (1) flexible Sprachen, die mindestens "one major class of multifunctional lexemes (V/N/A oder N/A)" aufweisen, und (2) "rigid"-Sprachen, "which only have one or more major classes of specialized lexemes (V-NA, V-N, or V) and languages at best show a strong tendency towards one of the types". Nach Rijkhoff (1992, 42f.) besitzen derartige Sprachen i. d. R. keine nomenspezifischen Kategorien wie Numerus und Genus, denn „flexible nouns are transnumeral“. Lewis $(1967,25 f$.$) bemerkt$ in diesem Zusammenhang: 'In Turkish nouns are both 'devoid of grammatical gender' and transnumeral or 'numerically neutral'”. Auch Kornfilt $(1997,265)$ konstatiert: "There are no different classes of nouns with respect to singular-plural marking”. Ein erster Hinweis für diese Art der Numerusverwendung findet sich bei Tobler (1883), dem zufolge nicht alle Nomen obligatorisch entweder nur als Singular oder Plural aufzufassen sind:

Wenn wir uns jedes Substantiv bei seiner Bildung unwillkürlich als Singular denken, so ist dieses eben damit nicht zugleich im Gegensatz zum Plural gedacht und es ergibt sich zunächst nur, daß der in diesem Namen ausgedrückte Gegensatz nicht ursprünglich, überdies der Name Singularis nicht glücklich gewählt ist. [...] Kurz: der sog. Singular ist ursprünglich Einheit des Begriffs, nicht der Zahl (Tobler, 1883, 410f.; zit. nach Biermann, 1982, 230).

In ähnlicher Weise argumentiert Wackernagel (1920, 84f) und macht auf diesen allgemeinen Charakterzug des Substantivs aufmerksam: „Aber an und für sich ist der Begriff eines Substantivs gegenüber dem Unterschied des Numerus indifferent. Wenn wir aus einem Paradigma den Stamm herausschälen, müssen wir ihm sowohl pluralische als singularische Bedeutung zugestehen. Dem Stamm eines Nomens haftet also an und für sich die singularische Bedeutung nicht an“ (Wackernagel, 1920, 84f; zit. nach Biermann, 1982: 230). Nach Grönbech (1936, 59) sind Nomen bereits in den frühen Schriftdokumenten des Türkischen als „Namen von Kategorien“ bzw. „Konzepten“ charakterisiert, und nicht als Ausdrücke, die auf individuellen Entitäten referieren:

Was ein jedes türkisches Nomen, mit Ausnahme der Eigennamen, bezeichnete, war also das Genus. at bezeichnete nicht an erster Stelle ein Pferd oder viele Pferde, sondern das Pferd als Begriff, die Gattung Pferd, alle denkbaren Pferde. Wir Europäer reden in Wörtern, die entweder den Einer oder eine Mehrheit von Einern ausdrücken, und in einigen Fällen denken wir dabei an die Gattung; der Türke sprach von der Gattung, und ob dieselbe in dem gegebenen Fall mehr oder weniger zahlreich vertreten war, kommt meist nicht zum Ausdruck (Grönbech, 1936, 59).

Auf dieser Basis lässt sich mit Götz (1980) eine weitere Annahme zum Ausdruck der Quantität im Türkischen ableiten: „Innerhalb des türkischen Numerusparadigmas bezeichnet der Singular die Stammform des Substantivs als hinsichtlich der semantischen Unterscheidung in Gattungs- 
und Individualbezeichnung in bestimmten Kontexten als merkmallose Form“ (Götz, 1980, 384). Ausdrücke, die keine Pluralendungen tragen, sind ihrer Bedeutung nach vage bzw. ambig zwischen einer singularischen und generischen Lesart, wenn nicht der Kontext die eine oder die andere Interpretation ausschließt. Greenberg (1972) stellt in diesem Zusammenhang fest:

A considerable number of classifier languages (e. g. many Iranian and Turkic languages, Korean) have what are generally described as plural affixes. However, closer examination seems to show that in almost every instance the «unmarked» singular is in fact a form which, like the collective in languages with a compulsory plural, is non-committal in regard to number (Greenberg, 1972, 30).

Die türkische Sprache gehört demnach dem Typus der Numerusverwendung an, die Corbett (2000) zufolge "general number" aufweisen. In dieser Art der Numerusverwendung kann die Bedeutung eines Nomens entweder ohne Bezug auf die Kategorie Numerus, d. h. mithilfe von Transnumeralia ausgedrückt werden, "[whereby] the whole category of number [...] is optional (the choice is whether to express number or not)" (Corbett, 2000, 50) oder die Bedeutung eines Nomens wird innerhalb des Numerussystems auf der Basis verschiedener Numeri realisiert. Im Türkischen besteht die Möglichkeit zwischen Singular und Plural zu wählen:

The system of marking number is obligatory wherever number marking is possible (with the exception of nonreferential nouns, where plurality marking is optional if the noun is bare, i.e. if there is no determiner). In other words, there are syntactic contexts where, despite plural semantics of the noun phrase, the head noun cannot be marked for plurality. When the noun phrase has numeral modifiers or certain quantifiers as modifiers, the plural suffix cannot occur (Kornfilt, 1997, 265).

Betrachten wir einige Verwendungen, z. B. beş çocuk [fünf Kind] „fünf Kinder“, birçok çocuk [viel/mehrere Kind] „viele/mehrere Kinder“ (Lewis, 1975, 75f.; "birçok 'a good deal of' is followed by a noun in singular or plural; pedants say singular only"). Bei einigen Quantifikatoren ist das Pluralsuffix jedoch obligatorisch, z. B. bazı çocuk-lar [manche Kind-PL] „manche Kinder“, bütün çocuk-lar [alle Kind-PL] „alle Kinder“, wie auch Kornfilt (1997, 266) feststellt: "Thus where plural marking is possible, the marking is obligatorily present. However, if the noun phrase lacks a determiner and is also nonreferential, plurality marking is optional". Wie lassen sich indes folgende Beispiele erklären?

13) »Kuş insan dilinden anlar mı?«.

[Vogel Mensch Sprache-POSS-ABL verstehen-PRÄS QUE] (Kemal, 1978, 22).

„Ob die Vögel die Sprache der Menschen verstehen?“ (Bischoff, 1984, 21).

14) Küçücük üç başparmak iriliğinde bir kuş, som mavi... (Kemal, 1978, 8).

[Klein-SUF-DIMIN drei Daumen groß-SUF-ADVER ein Vogel rein blau ...].

„Ein winziger Vogel, drei Daumen groß, von reinstem Blau ...“ (Bischoff, 1984, 8).

15) «Işste kuşlar buna dayanamaz,» dedi Süleyman (Kemal, 1978, 22).

[Genau Vogel-PL dies mögen-3P.-NEG sagen-PRÄT-1.SG Süleyman].

»Genau das mögen die Vögel gar nicht«, bemerkte Süleyman« (Bischoff, 1984, 21).

In Beispiel (13) stellt kuş die transnumerale Form dar, mit der Bedeutung „einen oder mehrere Vögel“. Im Falle der Referenz auf einen Vogel bzw. einen bestimmten Vogel, wird der Singular 
bir kuş wie in (14) verwendet, der Plural wird mithilfe des Suffixes -lAr gebildet, wie im Beispiel (15) dargestellt. Somit ergibt sich nachstehender Numeruskontrast:

Kuş gördüm [Vogel sehen-PRÄT.1.P.SG] (transnumeral: einen oder mehrere).

Bir kuş gördüm [Einen Vogel sehen-1.PRÄT.1.P.SG] (Singular: genau einen).

Kuşlar gördüm [Vogel-PLsehen-1.PRÄT.1.P.SG] (Plural: mehr als einen/Verschiedene).

Im Gegensatz zu Corbetts (2000) Begriffsbestimmung, die auf dem Kriterium [ \pm Nomen] basiert, definiert Gil (1996) Transnumeralität als "unmarked with respect to the mass/singular/ plural distinction” (Gil, 1996, 55; zit. n. Bliss, 2004, 4) und führt folgendes Beispiel aus dem Vietnamesischen an:

16) Su’ăn táo.

[Su essen Apfel].

„Su hat Apfel/einen Apfel/Äpfel gegessen“.

Nach Gil $(1996,55)$ ist das Nomen táo in Bezug auf die Individual-/Massennomen-Distinktion und Pluralnomen nicht markiert, d. h. táo kann als Massennomen, Singular oder Plural interpretiert werden (vgl. Bliss, 2004, 5). Sehen wir uns dazu ein ähnliches Beispiel aus dem Türkischen an:

17) Aylin elma ye-di.

[Aylin Apfel essen-PRÄT].

?, Aylin hat einen Apfel/Äpfel/Apfel gegessen“.

Hier kann elma singularisch („,ein Apfel“), pluralisch („Äpfel“) oder transnumeral als Massennomen interpretiert werden. Allerdings sind diese Lesarten im Falle bestimmter Nomen nicht möglich:

18) Selma zengin doktor bul-mak ist-iyor.

[Selma reich Arzt finden-INF wollen-PROGR].

„Selma will einen reichen Arzt/reiche Ärzte finden“.

Zengin doktor lässt sich entweder singularisch (,ein reicher Arzt“) oder pluralisch (,reiche Ärzte") interpretieren. Eine Lesart im Sinne einer Masse (,*reich-Arzt-Masse“) ist nicht möglich (Bliss, 2004, 5; vgl. Zwicky/Sadock (1975), "Ambiguity texts and how to fail them" und Cruse (1986), Lexical Semantics). Auch im Falle von elma, das theoretisch als Masse interpretiert werden kann, ist eine derartige Lesart eher nicht die Regel (Bliss, 2004, 5). Im Türkischen verhält sich die Mehrzahl der Nomen wie im Beispiel unter (18), d. h. ein Nomen kann (den Kontexten entsprechend) nicht gleichzeitig singularische, pluralische und transnumerale Lesarten aufweisen (ebd.). Da eine Interpretation von Nomen wie doktor als „Massekonzept“ (ebd.) nicht akzeptabel ist, lässt sich davon ausgehen, dass sich "bare nouns" im Türkischen transnumeral bzw. numerusindifferent verhalten. Außer der bereits angesprochenen Problematik birgt Gils (1996) Definition noch einen weiteren Nachteil. Gil (1996) vertritt die Ansicht, dass ein Nomen wie fish, z. B. Jimmy ate fish, ambig zwischen einer transnumeralen, singularischen und pluralischen Lesart ist (Gil, 1996: 5); zit. nach Bliss, 2004, 5f.). Allerdings gehört fish nicht demselben Typus von Nomen an, wie z. B. táo in (18), das transnumeral ist und nur eine Lesart zulässt (Bliss, 2004, 5f.). Die Numerusverwendung im Türkischen basiert somit auf folgendem Kontrast:

Languages of this type have an opposition general/singular versus plural [...] It does not follow that a language of this type cannot make a full 
number contrast. There are various possible means for making the distinction: use of numerals, especially 'one', use of an article (often the grammaticalization of the word for 'one'), and so on. But in such languages, the distinction is made 'when it matters' and not automatically, as in languages like English. The conditions, 'when it matters', to specify number, vary from language to language. The following characteristics may favour specifying number: being the topic as opposed to non-topic, first mention versus subsequent mention, referential versus non-referential use, human versus non-human, definite versus indefinite (Corbett, 2000, 14).

Die „lexikalische Sensitivität für Kontinuativität vs. Individuativität“ kommt im Türkischen in der „Kombinierbarkeit etwa mit dem unbestimmten Artikel zum Ausdruck“ (Zifonun, 2004, 409; Anmerkung: Ausführungen beziehen sich auf das Ungarische), z. B. bir muz. Nach Behrens (1995, 95f.) ist „in Bezeichnungen für größere Früchte [...] der individuative Aspekt der Geformtheit (als Einzelobjekt) lexikalisch angelegt“ (zit. nach Zifonun, 2004: 410), z. B. Bana (büyük) bir karpuz ver! „Gib mir eine (große) Melone!“. Im Gegensatz dazu sind mercimek „Linse“, fasulye „Bohne“, bezelye „Erbse“, buğday „Weizen“ und misır „Mais“ im Türkischen „kollektiv-kontinuativ“ bzw. transnumeral geprägt (Zifonun, 2004, 410; Anmerkung: Ausführungen beziehen sich wiederum auf das Ungarische). In diesem Zusammenhang heißt es bei Zifonun (2004):

Es ist anzunehmen, dass auch hier die Belebtheitsskala eine Rolle spielt: Gegenstände, die in der Belebtheitshierarchie einen vergleichsweise niedrigeren Rang einnehmen (materielle Gegenstände, Pflanzen, Früchte) können ggf. durch Kontinuativa bezeichnet werden, wobei perzeptive Faktoren (Kleinheit und Gleichförmigkeit der Einzelentitäten), [...], eine Rolle spielen mögen (Zifonun, 2004, 410f.; Vgl. Fussnote).

Nach Dahl $(2000,106)$ werden Menschen oder Tiere als stärker individualisiert empfunden (zit. nach Mihatsch, 2000, 47). Zum Türkischen "splitting feature: \pm animate" stellt Smith-Stark (1974) mit Verweis auf Lewis $(1967,106-107 ;$ 246-247) fest:

The traditional rule of grammar is that "inanimate plural subjects took a singular verb, plural verbs being used with animate subjects or with inanimates personified or considered as individuals." First and second persons agree in number with the verb. There are two sets of exceptions to the above general statement [...]. First, inanimates can take plural verbs if the distance between subject and verb is great. Second, factors of politeness allow the use of a plural verb with a singular third person subject as a sign of respect, and the substitution of first person plural for first person singular as a sign of modesty (Smith-Stark, 1974, 657- 671).

Darüber hinaus gibt es non-numerische Verwendungen des Plurals, d. h. Vagheit und Intensifikation, z. B. burada (wörtl. „,an diesem Ort“) „hier“, buralarda (wörtl. ,an diesen Orten“) „hier irgendwo“ (ähnlich engl. "hereabouts”) und uzakta „in der Ferne“, uzaklarda „in der weiten Ferne“; kapı yıkılıyordu „es wurde heftig an die Tür geklopft“, kapılar yıkllıyordu „es wurde sehr heftig an die Tür geklopft“ (Cruse, 1994, 2851), die den transnumeralen Aspekt in der türkischen Sprache verdeutlichen sollen. 


\section{Fazit und Ausblick}

Abschließend lässt sich mit Bassarak (1998) und Chierchia (1998) davon ausgehen, dass die türkische Sprache einen Mischtyp zwischen [NP[+arg, -pred]- und NP[+arg, +pred]-Sprachen ohne Artikel darstellt. Einerseits verhalten sich Nomen im Türkischen wie in [NP[+arg, -pred]Sprachen, d. h. sie sind transnumeral. Zur Aussonderung von distinkten Entitäten werden in Numeralkonstruktionen Klassifikatoren (fakultativ) herangezogen. Andererseits weist das Türkische auch Pluralformen auf, die Aspekte der Sortigkeit/Verschiedenheit beinhalten. Wie NP[+arg, +pred]-Sprachen ohne Artikel, verfügt die türkische Sprache über keine definiten Artikel. Zum Ausdruck der Definitheit werden andere Verfahren u. a. Numerus, Kasus und Demonstrativ herangezogen. Das übergreifende Prinzip in den grammatischen Ausdrucksformen der türkischen Sprache bildet somit das Konzept der Ökonomie.

\section{BIBLIOGRAPHIE}

Bassarak, A. (1987). "Numeralität und Transnumeralität im Türkischen”. In: W. U. Wurzel (Hrsg.) Studien zur Morphologie und Phonologie II. Akademie der Wissenschaften der DDR, Zentralinstitut für Sprachwissenschaft, Linguistische Studien. Reihe A, Arbeitsberichte Nr. 156, 1-32.

Bassarak, A. (1998). "Functional categories in Turkish - remarks on the interaction between morphology and syntax". In: Johanson, Lars (Hg.) The Mainz Meeting. Proceedings of the Seventh International Conference on Turkish Linguistics, August 3-6, 1994. Turcologica. Band 32. Wiesbaden: Harrassowitz Verlag, 45-56.

Behrens, L. (1995). "Categorizing between lexicon and grammar. The mass/count distinction in a crosslinguistic perspective.” In: Lexicology 1, 1-112.

Biermann, A. (1982). "Die grammatische Kategorie Numerus”. In: Hansjakob Seiler/C. Lehmann (Hrsg.). Apprehension. Das sprachliche Erfassen von Gegenständen. Teil I: Bereich und Ordnung der Phänomene. Tübingen: Narr, 229-243.

Bischoff, C. (1984). Auch die Vögel sind fort. Unionsverlag.

Bozkurt, F. (2007). Türkçenin Gizemi. Araştırma İnceleme Dizisi. İstanbul: Cem Yayınevi.

Chierchia, G. (1998). "Reference to Kinds Aacross Languages”. Natural Language Semantics, 6, 339-405.

Corbett, G. G. (2000). Number. UK. Cambridge University Press.

Cruse, D. A (1986). Lexical Semantics. UK. Cambridge University Press.

Cruse, D. A. (1994). "Number and Number Systems". Ed. In: Asher. Vol. 5, 2857ff.

Csató, É. A. (1998). “Turkish”. Eds. In: Johanson, Lars/ É. A. Csató. The Turkic Languages, 203-235. (Language Family Descriptions) London: Routledge.

Dahl, Ö. (2000). "Animacy and the Notion of Semantic Gender". In: Barbara Unterbeck/ Matti Rissanen (Eds.): Gender in Grammar and Cognition, 2 Vol. I: Approaches to Gender, II: Manifestations of Gender, 99-115. Berlin/ New York: Mouton de Gruyter.

Dogan, A. (1983). Sözcük Türleri. Türk Dil Kurumu Yayınları. Ankara: Olgaç Basımevi.

Ediskun, H. (1985). Türk Dilbilgisi. İstanbul: Remzi Kitabevi.

Gil, D. (1996). "Maltese 'collective nouns"': A typological perspective“. In: Rivista di linguistica, 8, 53-57.

Götz, M. (1980). „Singular und Plural im Türkeitürkischen“. In: Zeitschrift der Deutschen Morgenländischen Gesellschaft. Supplement IV. XX. Deutscher Orientalistentag vom 3. bis 8. Oktober 1977 in Erlangen, 382-385. Wolfgang Voigt (Hg.). Wiesbaden: Franz Steiner.

Greenberg, J. H. (1972). "Numeral Classifiers and Substantival Number: Problems in the Genesis of a Linguistic Type". In: Proceedings of the $11^{\text {th }}$ International Congress of Linguistics. Vol. 1, 17-37. Bologna-Florence. Aug. 28-Sept., 1972. Ed. Luigi Heilmann. Bologna: Società editrice il Mulino.

Grönbech, K. (1936). Der Türkische Sprachbau. Kopenhagen : Levin \& Munksgaard,

Grönbech, K. (1979). The Structure of the Turkic Languages. (Indiana University Uralic and Altaic series). Indiana University Research Institute for Inner Asian Studies. 
Hengeveld, K. (1992b). Non-verbal predication: Theory, typology, diachrony. Berlin: Mouton de Gruyter. Johanson, L., \& Rehbein, J. (Hrsg). (1999). "Probleme des Vergleichs Türkeitürkisch-Deutsch”. In: Türkisch und Deutsch im Vergleich. Wiesbaden: Harrassowitz Verlag.

Krifka, M. (1989). Nominalreferenz und Zeitkonstitution. Zur Semantik von Massentermen, Pluraltermen und Aspektklassen. München: Fink.

Krifka, M. (1991): „Massennomina“. In: Semantik. Ein internationales Handbuch der zeitgenössischen Forschung, 399-417. Berlin. New York: de Gruyter.

Leisi, E. (1971). Der Wortinhalt. Seine Struktur im Deutschen und Englischen. Heidelberg.

Lewis, G. (1967). Turkish Grammar. Oxford: Clarendon Press.

Meier, G. (2003). Orhan Pamuk Istanbul. München: Carl Hanser Verlag.

Pamuk, O. (2004). Istanbul. Yapı Kredi Yayınevi, İstanbul.

Riemann, W. (2007). Halid Ziya Uşaklıgil. Verbotene Liebe. Zürich: Unionsvelag.

Rijkhoff, J. (2002). The Noun Phrase. Oxford University Press. New York.

Smith-Stark, C. (1974). "The plurality split". In: Chicago Linguistics Society Papers, 10, 657-671.

Schröder, Chr. (1999). The Turkish Nominal Phrase in Spoken Discourse. Wiesbaden: Harrassowitz Verlag. Turcologica (Hrsg.) Lars Johanson. Band 40.

Tobler, L. (1883). "Über den Begriff und besondere Bedeutung des Plurals bei Substantiven." In: Zeitschrift für Völkerpsychologie und Sprachwissenschaft, 14, 410-434.

Uşaklıgil, H. Z. (1987). Aşk-ı Memnu (Yasak Aşk). İstanbul: Inkılâp Kitabevi.

Wackernagel, J. (1920). Vorlesungen über Syntax. Erste Reihe. Basel: Kommissionsverlag Emil Birkhäuser und Cie.

Yaşar, K. (1978). Kuşlar da Gitti. İstanbul: Ağaoğlu Yayınevi.

Zifonun, G. (2004). „Plural und Pluralität im Sprachvergleich, insbesondere zwischen dem Deutschen und Ungarischen“. In: D. Czicza, I. Hegedűs, P. Kappel, \& A. Németh (Hrsg.) (2004). Wertigkeiten, Geschichten und Kontraste, 397-415. Festschrift für Péter Bassola zum 60. Geburtstag. Szeged: Grimm Kiadó.

Zwicky, A., Jerrold, M., \& Sadock, M. (1975). "Ambiguity Tests and How to Fail Them". In: Syntax and Semantics 4, 1-36.

\section{Internetquellenverzeichnis}

Banguoğlu, Tahsin (1986). Türkçenin Grameri. Atatürk Kültür, Dil ve Tarih Yüksek Kurumu.

Türk Dil Kurumu Yayınlari: 528.

http://ebitik.azerblog.com/anbar/4441.pdf.

Bliss, Heather (2004). "The Semantics of the Bare Noun in Turkish". University of Calgary. Calgary Papers in Linguistics 25 (1). http://www.ucalgary.ca/lingcpl/volume25_1.html.

Görgülü, Emrah (2012). Semantics of Nouns and the Specification of Number in Turkish. Simon Fraser University. ega5@sfu.ca. Diss. http://www.sfu.ca/ ega5/Semantics\%20of\%20Nouns\%20and\%20the \%20Specification\%20of\%20Number\%20in\%20Turkish_Emrah\%20Gorgulu.pdf.

Mihatsch, Wiltrud (2000). „Wieso ist ein Kollektivum ein Kollektivum. Zentrum und Peripherie einer Kategorie am Beispiel des Spanischen“. In: PhiN 13/2000, 39-72. web.fu-berlin.de/phin/phin13/ p13t3.htm. 